[0212-7199 (2008) 25: 5; pp 241-243] ANALES DE MEDICINA INTERNA Copyright (C) 2008 ARAN EDICIONES, S.L.

AN. MED. InTERNA (Madrid) Vol. 25, N. $^{\circ} 5$, pp. $241-243,2008$

\title{
Tumores germinales mediastínicos
}

\author{
V. M. DÍAZ MUÑOZ DE LA ESPADA, P. KHOSRAVI SHAHI, B. HERNÁNDEZ \\ MARÍN, S. ENCINAS GARCÍA, J. A. ARRANZ ARIJA, G. PÉREZ-MANGA ${ }^{1}$ \\ Servicio de Oncología Médica. Hospital General Universitario Gregorio Marañón. Madrid
}

\author{
MEDIASTINAL GERM-CELL TUMOURS
}

\begin{abstract}
RESUMEN
Los tumores de células germinales del varón habitualmente se originan en los testículos. Sin embargo, en el 2-5\% de los casos pueden aparecer de forma primaria en localizaciones extragonadales, sin evidencia de un tumor testicular. Esta infrecuente entidad suele aparecer en la línea media corporal, predominántemente en el mediastino y en el retroperitoneo. Los tumores germinales extragonadales mediastínicos (TGEM) deben incluirse en el diagnóstico diferencial de cualquier tumor mediastínico de origen desconocido. Un diagnóstico exacto es fundamental, debido a que son tumores potencialmente curables con quimioterapia. En este artículo se revisan las características histopatológicas y clínicas de los TGEM, y sus diferencias los tumores germinales de origen testicular.
\end{abstract}

PALABRAS CLAVE: Tumores germinales. Extragonadal. Mediastínico.

\begin{abstract}
Germ-cell tumours of male ussually arise from the testis. However, in $2-5 \%$ of the cases, they also occur outside of the testis as a primary site without evidence of testicular primary tumour. This infrequent entity often appears in the body midline, predominantly in mediastinum and retroperitoneum. Mediastinal germ-cell tumours (MGCT) shall be included in the differential diagnosis of any mediastinic tumour of unknown origin. An accurate diagnosis is essential, due to the fact that these tumours are curable with chemotherapy. The histopathologic and clinical features, and its differences with germ-cell tumours from testicular origin are revised in this article.
\end{abstract}

KEY WORDS: Germ-cell tumours. Extragonadal. Mediastinal.

Díaz Muñoz de la Espada VM, Khosravi Shahi P, Hernández Marín B, Encinas García S, Arranz Arija JA, Pérez-Manga G. Tumores germinales mediastínicos. An Med Interna (Madrid) 2008; 25: 241-243.

\section{INTRODUCCIÓN}

Los tumores germinales extragonadales (TGE) constituyen solo el 2-5\% de los tumores de células germinales. Suelen originarse en las estructuras de la linea media corporal, sobre todo en el retroperitoneo y en el mediastino. Aún no se conoce con detalle el mecanismo por el cual aparece un tumor de células germinales fuera de las gónadas, sin evidencia de un tumor primario testicular. Aunque los tumores germinales extragonadales mediastínicos (TGEM) comparten muchas de las características propias de los tumores germinales de origen testicular, tienen algunas particularidades clínicas diferenciales. Los TGE mediastínicos tienen una especial incidencia en pacientes afectos del síndrome de Klinefelter (cariotipo XXY) y en su evolución tienen un especial riesgo de desarrollar neoplasias hematológicas. Además es un subtipo de tumores germinales con peor pronóstico que los tumores de origen testicular. Esta infrecuente entidad debe incluirse en el diagnóstico diferencial de cualquier neoplasia con aparición en forma de masa mediastínica. El diagnóstico exacto de esta neoplasia es fundamental, debido a que es potencialmente curable con quimioterapia.

\section{EPIDEMIOLOGÍA}

Los tumores de células germinales se originan generalmente en los testículos y se presentan principalmente en varones de 15-35 años. Sin embargo, un 2-5\% de los casos aparecen de forma primaria fuera de las gónadas, sin evidencia de un tumor primario testicular (1). Las localizaciones mas frecuentes de los tumores germinales extragonadales (TGE) son el mediastino y el retroperitoneo. Los TGE mediastínicos son los mas frecuentes y constituyen el 55\% de los tumores germinales extragonadales, apareciendo como grandes masas en el mediastino anterior (7). En la literatura se han descrito casos de tumores germinales primarios en múltiples localizaciones como la glándula pineal, próstata, cerebro o el hígado, aunque su aparición en estas localizaciones es excepcional $(2,3)$.

\section{HISTOGÉNESIS}

Durante años se ha cuestionado la existencia de los tumores germinales extragonadales, debido a que, en gran parte de los casos, estos pacientes presentaban anomalías testiculares

Trabajo aceptado: 18 de enero de 2008

Correspondencia: Víctor Manuel Díaz Muñoz de la Espada. Hospital General Universitario Gregorio Marañón. C/ Dr. Esquerdo, 46. 28007 Madrid. e-mail: vdm1978@hotmail.com 
sugerentes de la presencia de un tumor primario testicular oculto. El $30 \%$ de los pacientes con tumores germinales extragonadales presentan en los testículos signos sugerentes de la presencia de un tumor testicular primario que involucionó espontaneamente por mecanismos aún no bien conocidos (fenómeno de burned-out) (4). En otro 30\% de los pacientes con TGE se ha conseguido demostrar la presencia de carcinoma testicular in situ no invasivo (5), el cual tiene un alto riesgo de originar en su evolución tumores invasivos con capacidad metastática. Ante estos hallazgos algunos autores han sugerido que los tumores germinales extragonadales (TGE) son sólo la presentación metastática de un tumor testicular oculto o que ha involucionado espontaneamente (6). Sin embargo, estas anomalías testiculares no aparecen en todos los casos de TGEM. Por otra parte, los TGEM presentan algunas características clínicas e histopatológicas diferentes a los tumores germinales metastáticos de origen testicular, como son un peor pronóstico, mayor resistencia a la quimioterapia y una mayor frecuencia de aparición de neoplasias hematológicas en su evolución. Debido a esto, actualmente se acepta la existencia de los tumores germinales mediastínicos como una entidad independiente de los tumores de origen testicular.

Existen varias hipótesis sobre el origen de los TGEM. En estudios histopatológicos se han aislado células de estirpe germinal en múltiples órganos sanos como el hígado, médula ósea o cerebro, sin que aún se conozca con certeza su función (7). Algunos autores sugieren que los TG extragonadales podrían originarse a partir de estos grupos celulares. Los pacientes supervivientes de un TGEM tienen un riesgo del $10 \%$ de desarrollar un tumor testicular metacrónico a los 10 años de seguimiento (8), generalmente de estirpe histológica distinta a la del TGEM previo. Este hecho sugiere que los pacientes afectos de un TGEM pueden tener una alteración en su linea celular germinal que los predispone a la aparición de neoplasias germinales. Finalmente, el análisis genético de los TGE infantiles mostró un patrón similar al de las células germinales embrionarias (9). La teoría mas aceptada actualmente explica el origen de los TGEM a partir de células germinales embrionarias que han quedado distribuídas por las estructuras de la línea media corporal por un defecto en su migración durante la embriogénesis, desde la cresta urogenital hasta su localización definitiva en el testículo.

\section{DIAGNÓSTICO}

Esta entidad debe sospecharse ante toda masa mediastínica de apariencia tumoral en un paciente joven. Estos pacientes suelen presentarse con síntomas de ocupación mediastínica como tos, disnea o dolor torácico. Menos frecuentemente debutan con síndrome de vena cava superior, hemoptisis o disfagia.

La biopsia de la masa es el procedimiento diagnóstico de elección. El diagnóstico histológico preciso es fundamental, ya que con un correcto tratamiento de quimioterapia son tumores potencialmente curables, incluso en estadios avanzados. En algunos casos el análisis histologíco puede no ser concluyente, y ser indistinguible de otros tumores sólidos indiferenciados. En estos casos es útil la determinación cariotípica de la muestra. En el $90 \%$ de los tumores germinales se puede detectar la presencia de uno o varios isocromosomas p12 (copias del brazo corto del cromosoma 12) y la deleccción del brazo largo del cromosoma 12. Esta anomalía citogenética es muy específica de los tumores de estirpe germinal, tanto en tumores gonadales como extragonadales, y permite diagnosticar tumores germinales cuando el análisis histológico convencional no es capaz de llegar a un diagnóstico exacto. Por otra parte se ha demostrado que esta alteración cromosómica esta implicada directamente en la oncogénesis de estos tumores (10).

Al igual que los tumores de origen testicular, los TG mediastínicos pueden elevar marcadores tumorales séricos. El $80 \%$ de los de estirpe no seminomatosa elevan alfa-fetoproteína (AFP), beta-HCG o ambos. Los tumores del saco vitelino (yolk-sac), poliembriomas, carcinomas embrionarios y teratomas malignos suelen elevar AFP sérica, pero no beta-HCG. Los coriocarcinomas elevan beta-HCG, pero no suelen elevar AFP. Los seminomas puros no suelen elevar marcadores tumorales, aunque un 10-20\% de los casos tienen células del sincitiotrofoblasto gigante capaces de secretar beta-HCG. La presencia de AFP es incompatible con la presencia de un seminoma puro. Los niveles séricos de estos marcadores tumorales (AFP, beta-HCG y LDH) tienen valor pronóstico e implicaciones terapeúticas. Además permiten monitorizar la respuesta al tratamiento y detectar recidivas tumorales en el seguimiento de estos pacientes.

El estudio de extensión debe incluir un estudio ecográfico testicular para descartar la presencia de un tumor primario testicular y la realización de un TC toracoabdominal. El TC craneal solo está indicado en pacientes con criterios de mal pronóstico o por aparición de síntomas neurológicos. Se recomienda ofrecer criopreservación de semen a estos pacientes por el riesgo de esterilidad definitiva secundaria al tratamiento de quimioterapia.

\section{CARACTERÍSTICAS CLÍNICAS}

Los TGEM presentan la mayoría de las características propias de los tumores de células germinales testiculares. Son tumores malignos con capacidad metastática que suelen afectar a individuos menores de 40 años. Son tumores muy sensibles a la quimioterapia, y pueden ser curados con un tratamiento citostático adecuado en un alto porcentaje de los casos. Sin embargo, los TGEM tienen algunas características clínicas diferenciales. Se ha descrito una especial incidencia de TGEM en pacientes afectos de síndrome de Klinefelter (XXY). Se ha postulado que esta trisomía cromosomica puede contribuir a originar alteraciones genéticas en la linea celular germinal de estos pacientes que los predisponga a la aparición de estos tumores (11).

Los pacientes afectados de un TGEM tienen una especial incidencia de desarrollar una neoplasia hematológica en su evolución. Se estima que alrededor de un $6 \%$ de los pacientes afectados por un TGEM desarrollarán con el tiempo algún trastorno hematológico. Estos trastornos solo se han descrito en los TGEM de estirpe no seminomatosa, no en los TGEM de estirpe seminomatosa. Las patologías asociadas mas frecuentes son la leucemia megacarioblástica, mielodisplasia con anomalías megacariocíticas, trombocitosis esencial, leucemias mieloides agudas, e incluso se ha descrito algún caso de histiocitosis maligna y mastocitosis sistémica $(12,13)$. Estas neoplasias deben diferenciarse de las neoplasias secundarias al uso de los citostáticos empleados en los pacientes con TGEM. Las leucemias asociadas al uso de quimioterapia apa- 
recen habitualmente a los 5-7 años de finalizado el tratamiento, frecuentemente precedidas de un periodo preleucémico de mielodisplasia, y suelen progresar a una leucemia mieloide aguda, con frecuentes anomalías en los cromosomas 5, 7 y 11 . Sin embargo, las leucemias asociadas a los TGEM tienen una mediana de tiempo de presentación desde el diagnóstico del TGEM de tan solo 6 meses, incluso en el 30\% de los casos se presentan de forma simultanea al TGEM. Los blastos de las leucemias asociadas a los TGEM presentan isocromosomas p12 hasta en el 38\% de los casos. Además, en algunos casos tambien es posible identificar también la trisomía XXY en estas células. Debido a esto, algunos autores sugieren que las células leucémicas y las células tumorales de estirpe germinal podrían proceder de un precursor común genéticamente alterado del saco vitelino embrionario (14). Finalmente es importante destacar que el pronóstico de las neoplasias hematológicas asociadas a los TGEM es muy pobre, con una mediana de supervivencia de 5 meses, incluso con tratamiento intensivo de quimioterapia.

\section{PRONÓSTICO Y TRATAMIENTO}

El tratamiento de elección de los TGEM es la quimioterapia seguida de la resección de las masas residuales. Los esquemas de tratamiento son similares a los usados en los tumores germinales de origen testicular (15). Con quimioterapia basada en el cisplatino se consiguen remisiones completas a largo plazo en el 50\% de los casos (7). El esquema de quimioterapia mas frecuentemente empleado incluye 3-4 ciclos de cisplatino en combinación con etopósido y bleomicina (BEP). Cuando tras completar el tratamiento de quimioterapia, las pruebas de imagen revelan persistencia de masas residuales, suele indicarse la resección quirúrgica de las mismas cuando es técnicamente posible. Aunque en la mayoría de los casos de tumores germinales de origen testicular no existen

\section{Bibliografía}

1. Collins DH, Pugh RCB. Classification and frecuency of testicular cancer. Br J Urol 1964; 36: 1-11.

2. Manivel JC, Pambuccian S. Germ cell tumor-like neoplasm occurring outside the anatomic midline. Semin Diagn Pathol 2003; 20: 260-71.

3. Hart WR. Primary endodermal sinus (yolk-sac) tumor of the liver: First reported case. Cancer 1975; 35: 1453-8.

4. Fabre E, Jira H, Izard V, Ferlicot S, Hammoudi Y, Theodore C. "Burned-out" primary testicular cancer. Br J Urol 2004; 94: 74.

5. Daugaard G, von der Maase H, Olsen J, Rorth M, Skakkeback NE. Carcinoma -in situ- of the testis in patients with assumed extragonadal germ-cell tumours. Lancet 1987; 2: 528-30.

6. Scholz M, Zehender M, Thalmann GN, Borner M,Thonr H. Extragonadal retroperitoneal germ cell tumour: Evidence of origin in the testis. Ann Oncol 2002; 13: 121-4.

7. Friedman NB. The function of primordial germ cell in extragonadal tissue. Int J Androl 1987; 10: 43-96.

8. Bokemeyer C, Nichols CR, Droz JP, Schmoll HJ, Horwich A, Gerl A, et al. Extragonadal Germ Cell Tumors of the Mediastinum and Retroperitoneum: Results From an International Analysis. J Clin Oncol 2002; 20: 1864-73.

9. Schneider DT, Schuster AE, Fritsch MK, Hu J, Olson T, Lauer S. Multipoint imprinting analysis indicates a common precursor in gonadal and nongonadal pediatric germ cells tumors. Cancer Res 2001; 6: 7268-76.

10. Chaganti RSK, Houldsworth J: Genetics and biology of adult human male germ cell tumors. Cancer Res 2000; 60: 1475-82.

11. Nichols CR, Heerema NA, Palmer C, Klinefelter, S. Syndrome associated with mediastinal germ cell neoplasms. J Clin Oncol 1987; 5: 1290-4. células tumorales viables en estas masas residuales, hasta en un $30 \%$ de los TGEM se pueden observar restos tumorales viables en el análisis de la pieza de resección (7). En caso de elementos tumorales viables en las piezas de resección, aún no existen datos para recomendar sistemáticamente la administración de quimioterapia tras la cirugía, y la decisión suele individualizarse a cada caso concreto.

En general, los TGEM tienen peor pronóstico que los tumores germinales testiculares (16). En la clasificación pronóstica actual de los tumores germinales del Grupo Cooperativo Internacional de Tumores de Células Germinales (International Germ-Cell Cancer Collaborative Group -IGCCCG-), los TGEM no seminomatosos se incluyen directamente en el subgrupo de mal pronóstico. Aunque algunos autores han tratado de establecer criterios pronósticos específicos para los tumores germinales extragonadales, estos criterios están aún por validar en estudios prospectivos (17). Tras conseguir la remisión tumoral con la quimioterapia, hasta el $50 \%$ de los pacientes tratados de TGEM con quimioterapia recaerán de su enfermedad. El mayor riesgo de recaída se centra en los 2 primeros años tras la remisión, aunque se han descrito recaídas tardías y la aparición de tumores testiculares metacrónicos incluso a los 20 años de evolución. El seguimiento estricto de estos pacientes por tanto es fundamental. Cuando se produce una recaída o refractariedad al tratamiento inicial, los esquemas de quimioterapia de rescate se basan en drogas como el paclitaxel o la ifosfamida en combinación con cisplatino y pueden conseguir la curación en algunos de estos pacientes (18). En general la supervivencia a 5 años es solo del $50 \%$ en los casos de TEGM no seminomatoso, aunque puede llegar al $80 \%$ en los casos de TGEM de tipo seminoma puro, dependiendo del subgrupo de riesgo (7). Finalmente se debe recordar que el uso de quimioterapia a altas dosis seguido de transplante autólogo de médula ósea no conseguido mejorar sustancialmente el pronóstico de los pacientes refractarios a la primera linea de tratamiento en los estudios publicados, por lo que aún se considera un tratamiento experimental.

12. Roth BJ, Heerema N, Griep J, Tricot G. Hematologic neoplasia associated with primary mediastinal germ-cell tumors.N Engl J Med 1990; 322: $1425-9$.

13. Ladanyi M, Roy I. Mediastinal germ cell tumors and histiocytosis. Hum Pathol 1988; 16: 586-90.

14. Hartmann JT, Nichols CR, Droz JP. Hematologic disorders associated with primary mediastinal nonseminomatous germ cell tumors. J Natl Cancer Inst 2000; 92: 54-61.

15. Schmoll HJ, Souchon R, Krege S, Albers P, Beyer J, Kollmaniisberger $\mathrm{C}$, et al. European consensus on diagnosis and treatment of germ cell cancer: A report of the European germ Cell Cancer Consensus Group (EGCCCG). Ann Oncol 2004; 15: 1377-99.

16. Hartmann JT, Nichols CR, Droz JP et al. Prognostic variables for response and outcome in patients with extragonadal germ-cell tumors. Ann Oncol 2002; 13: 1017-28.

17. Hartmann JT, Nichols CR, Droz JP, Horwich A, Gerl A, Fossa SD, et al. Prognostic variables for response and outcome in patients with extragonadal germ-cell tumors. Ann Oncol 2002; 13: 1017-28.

18. Hartmann JT, Einhorn L, Nichols CR, Droz JP, Horwich A, Gerl A, et al. Second-Line Chemotherapy in Patients With Relased Extragonadal Nonseminomatous Germ Cell Tumors: Results of an International Multicenter Analysis. J Clin Oncol 2001; 19: 1641-8.

19. Bokemeyer C, Kollmannsberger C, Meisner C, et al. First-line high-dose chemotherapy compared with standard-dose PEB/VIP chemotherapy in patients with advanced germ cell tumors: A multivariate and matchedpair analysis. J Clin Oncol 1999; 17: 3450-6. 